\title{
Delocalized Lattice Plasmon Resonances Show Dispersive Quality Factors
}

\author{
Wei Zhou, ${ }^{\dagger}$ Yi Hua, ${ }^{\dagger}$ Mark D. Huntington, ${ }^{\dagger}$ and Teri W. Odom $*, \dagger, \ddagger$ \\ ${ }^{\dagger}$ Department of Materials Science and Engineering, Northwestern University, 2220 Campus Drive, Evanston, Illinois 60208, United \\ States \\ ${ }^{\ddagger}$ Department of Chemistry, Northwestern University, 2145 Sheridan Road, Evanston, Illinois 60208, United States
}

\begin{abstract}
This Letter describes how out-of-plane lattice plasmon (OLP) resonances in 2D Au nanoparticle (NP) arrays show dispersive quality factors. These quality factors can be tailored simply by controlling NP height. Numerical calculations of near-field optical properties and band diagrams were performed to understand the measured dispersion effects of the OLPs. The results revealed that delocalized OLPs are a type of surface Bloch mode composed of many Bloch harmonics. As the OLP dispersion evolves from a stationary state to a propagating state, the nonradiative loss decreases because of weak local field confinement, whereas the radiative loss increases because of strong coupling to the leaky zero-order harmonic.
\end{abstract}
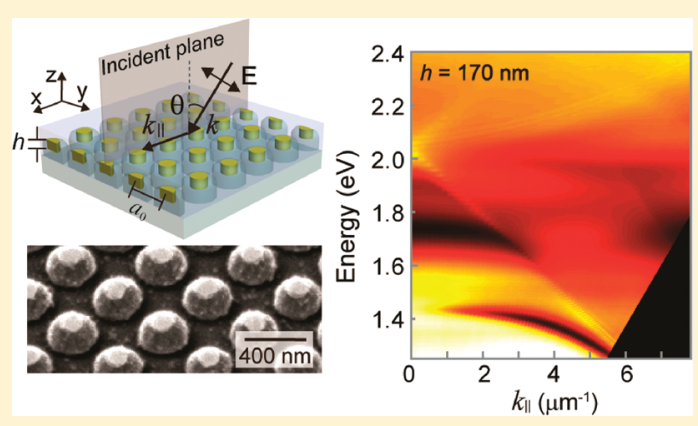

SECTION: Plasmonics, Optical Materials, and Hard Matter

S urface plasmon resonances supported in metal nanostructures can concentrate optical fields into nanoscale volumes, ${ }^{1,2}$ which is important for applications such as plasmonic nanolasers, ${ }^{3,4}$ optical modulators, ${ }^{5}$ chemical-biological sensors, ${ }^{6}$ and nonlinear optical devices. ${ }^{7,8}$ A slower depletion of plasmon energy is desirable to enhance local optical fields further and to achieve a narrow plasmon resonance line width. ${ }^{9}$ The damping of plasmons is characterized by the resonance quality factor $Q_{\text {tot }}(=\lambda / \delta \lambda=$ $\omega / \delta \omega)$, which is determined by both radiative (e.g., the electricdipole radiation) and nonradiative (e.g., electron-electron, electron-phonon, and electron-defect interactions) processes. ${ }^{10}$ For localized surface plasmon (LSP) resonances under the quasistatic condition (when the physical size of the resonator is small compared with local variations in the electromagnetic (EM) field), the nonradiative quality factor $Q_{\mathrm{nr}}$ has a theoretical upper limit of $\left(\mathrm{d} \varepsilon_{\mathrm{m}}^{\prime} / \mathrm{d} \omega\right) \omega / 2 \varepsilon_{\mathrm{m}}^{\prime \prime}$ ( $<20$ for $\mathrm{Au}$ in the optical range) because a major portion of the EM energy is stored and dissipated in the metal. ${ }^{11}$ Recent work has shown that plasmonic structures with broken symmetries support multipolar dark LSP resonances beyond the quasistatic limit, ${ }^{12-14}$ where both radiative and nonradiative losses are suppressed. These dark plasmon resonances cannot be easily tuned, however, without changing the overall geometry of the nanostructures.

Another strategy to achieve resonances with high $Q_{\text {tot }}$ is to take advantage of lattice plasmon resonances in periodic nanoparticle (NP) arrays, ${ }^{15-19}$ where the radiative loss is significantly reduced by dipolar interactions between NPs. In addition, because lattice plasmons are a type of delocalized plasmon resonance, $Q_{\mathrm{nr}}$ is not restricted to a theoretical upper limit for quasistatic LSPs. Because of challenges in fabricating NPs with heights $(h)>100 \mathrm{~nm}$, most work has focused on in- plane lattice plasmons that are mediated by strong coupling between the in-plane dipole moments of the NPs. ${ }^{15-19}$ The properties of in-plane lattice plasmons are typically described using the coupled dipole approximation (CDA) method; ${ }^{15,16}$ however, because CDA uses plane-wave excitation to determine the fields self-consistently, there are challenges in characterizing dark plasmons, which cannot be excited by free-space light. Recently, we reported a new type of lattice plasmon in $2 \mathrm{D}$ arrays of large NPs ( $>100 \mathrm{~nm}$ in all three dimensions) that is mediated by out-of-plane dipolar interactions. ${ }^{20}$ Out-of-plane lattice plasmons (OLPs) can be easily tuned by changing the height of the NPs in the array. Significantly, unlike in-plane lattice plasmons, ${ }^{19}$ OLPs can maintain a high resonance quality factor $Q_{\text {tot }}(>100)$ even when the dispersion relation is flatbanded. ${ }^{20}$

This Letter demonstrates that the $Q_{\text {tot }}$ of OLPs is highly dispersive and depends sensitively on the height of the NPs. Computational methods reveal that OLPs can be considered as a type of surface Bloch mode composed of many Bloch harmonics. As the OLP dispersion evolves from a stationary state to a propagating state, the nonradiative loss decreases because a smaller portion of optical energy is stored in the NPs; in contrast, the radiative loss increases because more efficient coupling occurs between higher order Bloch harmonics and the leaky zero-order harmonic. This system provides a framework for understanding the dispersive properties of delocalized plasmon resonances supported by other types of plasmonic nanostructures.

Received: March 15, 2012

Accepted: May 3, 2012

Published: May 3, 2012 
Large areas $\left(>1 \mathrm{~cm}^{2}\right)$ of $2 \mathrm{D}$ square arrays of $\mathrm{Au}$ NPs (periodicity $a_{0}=400 \mathrm{~nm}$, diameter $d=160 \mathrm{~nm}$ ) were fabricated using soft nanolithography and template stripping. ${ }^{20}$ To investigate the dependence of the OLP dispersion properties on NP height, we created a range of samples with different heights $(h=90,110,135,170 \mathrm{~nm})$. Zero-order reflection and transmittance spectra were measured under incident angles $\theta=$ 10 to $60^{\circ}$ using a home-built rotational stage, the in-plane wavevector $k_{\|}$along the $x$ direction (Figure 1A). We
A

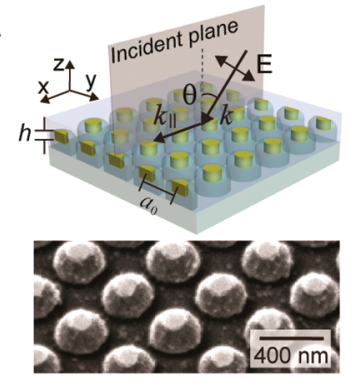

D

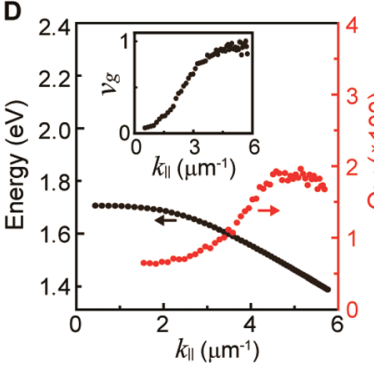

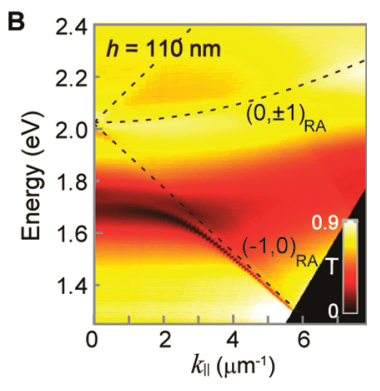

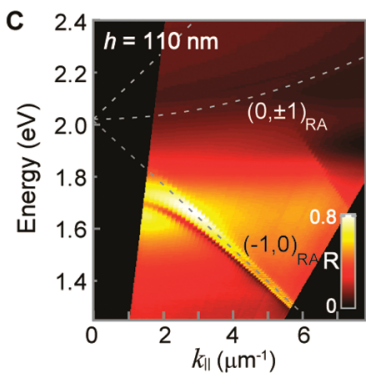

Figure 1. Out-of-plane lattice plasmons in 2D $\mathrm{Au}$ NPs exhibit wavevector-dependent resonance energies and quality factors. (A) Light excitation scheme under TM-polarized light and SEM image of $\mathrm{Au}$ NP arrays supported in PU at a tilted angle. (B) Measured transmittance $\left(\theta\right.$ from 0 to $60^{\circ}$ ) and (C) reflectance (from 10 to $60^{\circ}$ ) spectra as a function of in-plane wavevector $\left(k_{\|}=k_{0} \cdot \sin \theta\right)$ and energy $(E=\hbar \omega)$ for 2D Au NP arrays $(h=110 \mathrm{~nm}, d=160 \mathrm{~nm})$. (D) Measured resonance energy $E$ and quality factor $Q_{\text {tot }}$ of the out-ofplane lattice plasmons as a function of in-plane wavevector $k_{\|}$. (inset) Group velocity $\nu_{\mathrm{g}}$ normalized to the speed of light in the dielectric environment surrounding the NP arrays $(n=1.52)$ as a function of $k_{\|}$.

illuminated the samples by collimated TM-polarized ( $p$ polarized) white light from a halogen lamp (100 W) with a spot size of $2 \mathrm{~mm}$. The reflected or transmitted light was coupled to a bundled optical fiber connected to a CCD spectrometer (Triax 552/ $\mathrm{LN}_{2}-$ Cooled CCD, Horiba Jobin Yvon). All optical measurements were performed in indexmatched conditions, where PU substrate $\left(n_{\mathrm{PU}}=1.55\right)$ was in contact with an oil superstrate $\left(n_{\text {oil }}=1.525\right)$.

Figure 1B,C displays the measured transmittance and reflectance spectra as a function of in-plane wavevector $\left(k_{\|}=\right.$ $\left.k_{0} * \sin \theta\right)$ and energy $(E=\hbar \omega)$ for a Au NP array with $h=110$ $\mathrm{nm}$. Two types of resonances with different dispersion properties were observed: (1) a broad, in-plane plasmon resonance $\left(Q_{\text {tot }} \approx 8\right)$ centered at $E=1.7 \mathrm{eV}$, which can be attributed to individual $\mathrm{NPs}^{20}$ and (2) a narrow, dispersive OLP resonance, which is flat-banded at small $k_{\|}$and converges with the $(-1,0)$ Rayleigh anomaly $(\mathrm{RA})$ at large $k_{\|}$. At small $k_{\|}$, the OLP exhibits a low normalized group velocity $\left[v_{\mathrm{g}} /(\mathrm{c} / n)=\right.$ $\left.\left(\delta \omega / \delta \mathrm{k}_{\|}\right) /(\mathrm{c} / n)\right]$ (Figure 1D, inset) characteristic of a strong concentration of optical fields by plasmonic structures. ${ }^{19,21}$ As $k_{\|}$increases from 0 to $6 \mu \mathrm{m}^{-1}, v_{\mathrm{g}} /(\mathrm{c} / n)$ gradually increases from 0 to 1 as the stationary state becomes a propagating one (Figure 1D, inset). The $Q_{\text {tot }}$ of the OLPs can be determined by measuring the resonance linewidths in the reflectance and transmittance spectra; this value gradually increases from $\sim 60$ at $k_{\|}=1.5 \mu \mathrm{m}^{-1}$ to $\sim 180$ at $k_{\|}=5 \mu \mathrm{m}^{-1}$ (Figure 1D). This strong dependence of $Q_{\text {tot }}$ on wavevector $k_{\|}$indicates that the depletion time of stored plasmon energy is related to the dispersion properties of OLPs.

To understand the observed dispersion effects on the OLP resonances, we performed 3D finite-difference time-domain (FDTD) calculations to obtain the band diagrams and nearfield optical properties of $2 \mathrm{D}$ periodic Au NP arrays. ${ }^{22-25}$ One unit cell was modeled using a uniform mesh size of $3 \mathrm{~nm}(x, y$, and $z$ directions) with Bloch boundary conditions in the $x-y$ dimensions and a perfectly matched layer in $z$. We used a pulsed, point-dipole source, which can generate optical fields with a broad range of frequency and wavevectors in the nearfield, to excite OLPs across the entire $k$ range, even when they cannot be excited by free-space light. ${ }^{26}$ The dielectric constants of Au were included in the modeling by using a Lorentz-Drude model with parameters fitted to experimental data from 400 to $1000 \mathrm{~nm},{ }^{27}$ and $\mathrm{Au} \mathrm{NP}$ is modeled in a uniform dielectric environment $(n=1.525)$.

Figure 2A shows the FDTD-calculated band diagram of a 2D array of $\mathrm{Au} \mathrm{NPs}(h=110 \mathrm{~nm})$, which displays the $E-k$
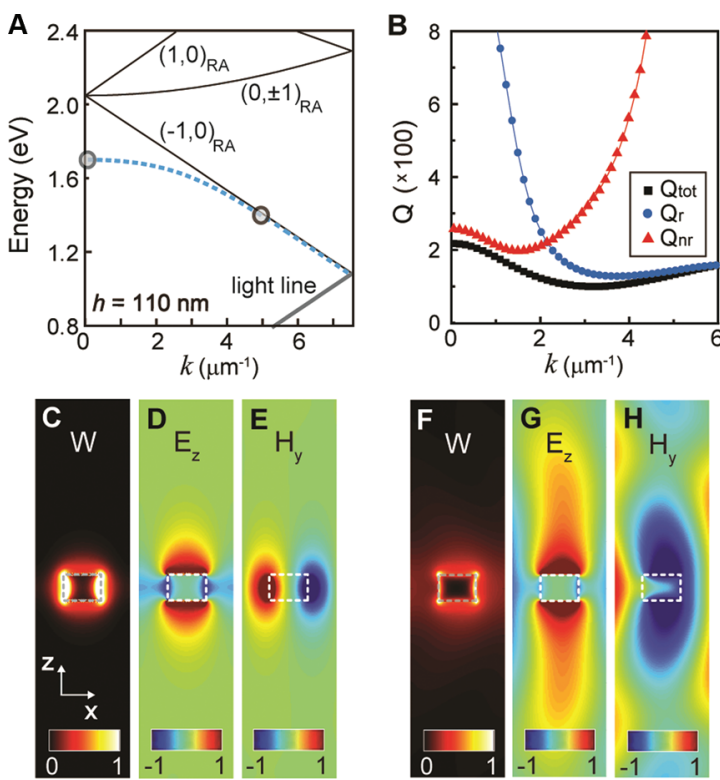

Figure 2. Optical energy of the out-of-plane lattice plasmon mode is concentrated in the Au NPs when the mode is a stationary state. (A) FDTD-calculated band diagram of 2D Au NP arrays $(h=110 \mathrm{~nm})$. (B) FDTD-calculated quality factors $Q_{\text {tot }}, Q_{r}$, and $Q_{\mathrm{nr}}$ of OLP mode as a function of $k .(\mathrm{C}-\mathrm{H})$ FDTD-calculated maps of energy density, $E_{z}$ and $H_{y}$ at $(\mathrm{C}-\mathrm{E})$ a stationary state $(k=0)$, and $(\mathrm{F}-\mathrm{H})$ a propagating state $\left(k=5 \mu \mathrm{m}^{-1}\right)$.

dispersion relations of various Bloch modes in the periodic structure. In addition to RAs associated with the different surface diffraction orders inherent in the $2 \mathrm{D}$ grating, the calculated band diagram contains a flat-banded dispersion curve above the light line that matches the one associated with the OLP mode in experiment (Figure 1D). Hence, the experimentally measured OLP resonances are a type of surface Bloch mode intrinsic to $2 \mathrm{D}$ periodic arrays of Au NP. These 
modes do not rely on coupling between a localized and delocalized mode; therefore, OLPs can maintain a narrow resonance line width even when their dispersion is flat-banded with no overlap with that of a diffraction mode. As a type of Bloch mode, lattice plasmons can be decomposed into Bloch harmonics: ${ }^{24,28} E_{k}(x, y, z)=\Sigma_{m} U_{k}^{m}(y, z) \mathrm{e}^{i\left((2 \pi m) /\left(a_{0}\right)+k\right) x}$, where $k$ is the fundamental wavevector along $x$ in the first Brillion zone, $a_{0}$ is the period, $m$ is the index of the Bloch harmonic, and $U_{k}^{m}(y, z)$ is the complex amplitude of each Bloch harmonic. On the basis of its dispersion relation (Figure $2 \mathrm{~A}$ ), the zero-order harmonic of the OLP mode lies above the light line $(\mathrm{I}(2 \pi m) /$ $\left.\left(a_{0}\right)+k \mid<n(\omega) /(c), m=0\right)$ and can radiate energy into free space as photons, whereas other higher order harmonics are below the light line $\left(I(2 \pi m) /\left(a_{0}\right)+k \mid>n(\omega) /(c), m= \pm 1, \pm 2\right.$, ...) and are evanescent modes bound to the plane of the NP arrays. Therefore, OLPs are a type of leaky surface Bloch mode. By reciprocity, OLPs can be excited by free-space light at oblique angles when the in-plane wavevector $k_{\|}$of incident light matches with the wavevector $k$ of the OLPs.

Compared with leaky Bloch modes in photonic crystal slabs where stored energy is dissipated only radiatively, ${ }^{22,23}$ the OLP modes also involve nonradiative loss because of its plasmonic nature. Therefore, to understand the dispersion in $Q_{\text {tot }}$, we need to investigate the radiative quality factor $Q_{r}$ and nonradiative quality factor $Q_{\mathrm{nr}}$ separately. From FDTD calculations, $Q_{r}$ can be determined by the line width of resonant spectra in "lossless" Au NP arrays, where the dielectric constants of Au are modified by setting the imaginary part of the permittivity to zero. $^{29} Q_{\mathrm{nr}}$ can then be calculated from the relation $Q_{\text {tot }}{ }^{-1}=$ $Q_{r}^{-1}+Q_{n r}{ }^{-1}$. Figure $2 B$ shows that the calculated $Q_{\text {tot }}$ decreases from $\sim 210$ to $\sim 100$ as $k$ increases from 0 to $3 \mu \mathrm{m}^{-1}$ and then increases to 150 as $k$ increases to $6 \mu \mathrm{m}^{-1}$, which is in qualitative agreement with the measured results (Figure 1D). After decomposing $Q_{\text {tot }}$ into $Q_{\mathrm{r}}$ and $Q_{\mathrm{nr}}$, we observed that each quality factor evolves very differently with $k$. At small $k(<1$ $\mu \mathrm{m}^{-1}$ ), the radiative loss is extremely small, and the nonradiative loss dominates the total loss. As $k$ increases from 0 to $2 \mu \mathrm{m}^{-1}, Q_{r}$ drastically drops from $>1000$ to $\sim 200$, whereas the change of $Q_{\mathrm{nr}}$ is small. As $k$ further increases from 2 to $6 \mu \mathrm{m}^{-1}, Q_{\mathrm{nr}}$ increases dramatically from $\sim 200$ to $>1000$, whereas the change of $Q_{r}$ is small.

A microscopic near-field optical picture is needed to understand the dispersion effects on $Q_{\text {tot }}$. Figure $2 \mathrm{C}-\mathrm{H}$ compares near-field optical properties as the dispersion evolves from a stationary state $(k=0)$ to a propagating state $(k=5$ $\left.\mu \mathrm{m}^{-1}\right)$. At the stationary state (Figure 2C), the EM energy density $W\left(W=(1 / 2)\left[\operatorname{Re}[(\mathrm{d}(\omega \varepsilon)) /(\mathrm{d} \omega)]|E|^{2}+\mu|H|^{2}\right]\right)$ map shows that a significant portion of the optical energy of OLPs is stored inside the Au NPs, which results in a dominant $Q_{n r}$. $E_{z}$ shows maxima with the same phase on the top and bottom of the NPs, which corresponds to an out-of-plane dipolar oscillation (Figure 2D) with an extremely small radiation in $z$. $H_{y}$ shows maxima with opposite phase on lateral edges of the NPs in $x-z$ cross-section, which indicates that a circulating magnetic field around the NPs is coupled to the alternating charge current driven by $E_{z}$ along the $z$ axis (Figure $2 \mathrm{E}$ ).

At the propagating state (Figure $2 \mathrm{~F}$ ), most of the optical energy is stored in the surrounding dielectric instead of in the metal, which leads to much smaller nonradiative losses compared with the stationary state. In addition, $E_{z}$ (Figure $2 \mathrm{G})$ and $H_{y}$ (Figure $2 \mathrm{H}$ ) are in-phase with a well-defined phasefront along the wavevector direction ( $x$ axis), characteristics that are similar to transverse EM waves in free space.
Compared with the stationary state, $E_{z}$ of the propagating state has a much larger penetration depth into the dielectric environment, which indicates a weaker local optical field confinement.

To demonstrate that the measured dispersion in $Q_{\text {tot }}$ is general for OLPs, we examined the optical properties of $2 \mathrm{D}$ arrays of Au NPs with different NP heights. Figure 3 shows that
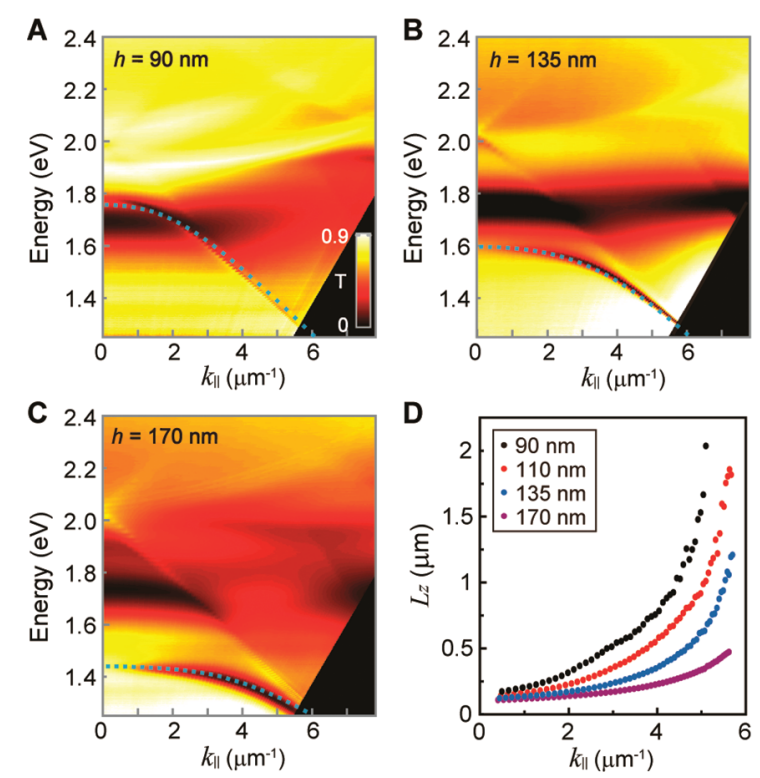

Figure 3. Dispersion properties of out-of-plane lattice plasmons significantly depend on NP height. Measured transmittance as a function of in-plane wavevector $k_{\|}$and energy $E$ in the form of dispersion diagrams for 2D Au NP arrays with NP heights of (A) 90, (B) 135, and (C) $170 \mathrm{~nm}$. The dashed curves correspond to the FDTD-calculated dispersion relations of OLP. (D) Estimated decay length $L_{z}$ in Au NP arrays with different NP heights confirms that OLPs exhibit tighter local field confinements at the stationary state, in agreement with FDTD-calculated $E_{z}$ distribution maps in Figure 2.

OLP modes can be continuously modified by changing NP height. In contrast, other spectral features, including the dispersionless in-plane plasmon resonances associated with individual NPs and RA surface diffraction modes, are not sensitive to changes in NP height. As the height increases from $h=90$ to $170 \mathrm{~nm}$, the resonance energy of OLPs at the stationary state $\left(k_{\|}=0\right)$ decreases from 1.78 (Figure $3 \mathrm{~A}$ ) to $1.45 \mathrm{eV}$ (Figure 3C) because of the longer oscillation pathlength defined by the NP height. ${ }^{1}$ Also, the dispersion of the OLPs in NP arrays with smaller $h$ converges with the $(-1,0)$ $\mathrm{RA}$ at smaller $k_{\|}$. Overall, the measured dispersion relations of OLPs for NP arrays with different heights matched well with the FDTD-calculated dispersion relations (dashed curves).

As a type of surface Bloch mode, OLPs can confine EM fields to the plane of the NP array. The decay length $L_{z}$ can be approximated by the first-order harmonic component bound to the surface: ${ }^{19} L_{z}=1 /\left(2 * \operatorname{Im}\left(k_{z}\right)\right) ; k_{z}^{2}+\left(k_{\|}-2 \pi / a_{0}\right)^{2}=(n \cdot \omega /$ $c)^{2}$. Importantly, $L_{z}$ can be determined from the measured $E-$ $k_{\|}$dispersion relations. Figure 3D shows that $L_{z}$ increases from $150 \mathrm{~nm}$ at the stationary state $\left(k_{\|} \approx 0 \mu \mathrm{m}^{-1}\right)$ to $1 \mu \mathrm{m}$ at the propagating state $\left(k_{\|} \approx 5 \mu \mathrm{m}^{-1}\right)$ for NP arrays with $h=110$ $\mathrm{nm}$. This result confirms the main result of the FDTDcalculated $E_{z}$ distribution maps (Figure 2D,G), where the OLP exhibits a much tighter local field confinement at the stationary state. In addition, as the NP height increases, the rate of 
increase in $L_{z}$ with $k_{\|}$becomes slower because the OLPs approach the propagating state at larger $k_{\|}$.

Figure $4 \mathrm{~A}$ shows the dependence of $Q_{\text {tot }}$ extracted from measured optical spectra of OLPs in NP arrays with different
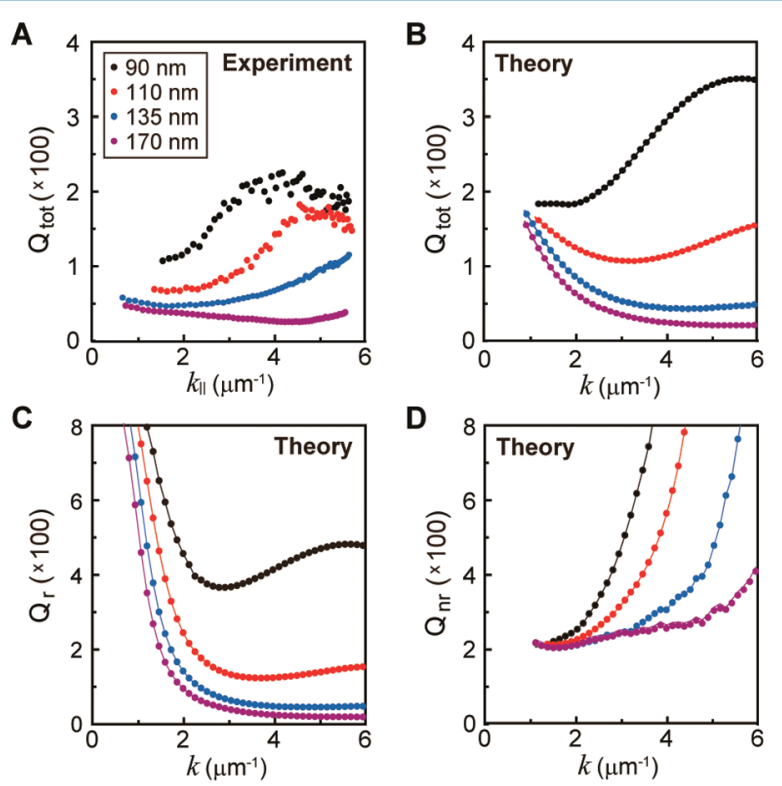

Figure 4. $Q_{\text {tot }}$ of out-of-plane lattice plasmons is dispersive and sensitive to changes in NP height. (A) Measured total quality factors $Q_{\text {tot }}$ and FDTD-calculated (B) $Q_{\text {tot }}$ (C) $Q_{r}$, and (D) $Q_{\text {nr }}$ show that changes in NP height not only influence $Q_{r}$ by determining coupling strength to the zero-order Bloch harmonic but also $Q_{\mathrm{nr}}$ by modifying the shape of dispersion relation of OLPs.

heights, on $k_{\|}$. Several features are apparent from these curves: (1) Over the entire $k_{\|}$range, NP arrays of smaller heights $(h=$ $90 \mathrm{~nm}, 110 \mathrm{~nm}$ ) tend to support OLPs with larger quality factors $Q_{\text {tot }}(>100)$; (2) at small $k_{\|}\left(<2 \mu \mathrm{m}^{-1}\right), Q_{\text {tot }}$ deceases as $k_{\|}$increases; and (3) at larger $k_{\|}\left(>2 \mu \mathrm{m}^{-1}\right), Q_{\text {tot }}$ starts to increase as $k_{\|}$increases, especially for NP arrays with smaller heights. FDTD-calculated curves of $Q_{\text {tot }}$ (Figure 4B) qualitatively match the experimental results. Compared with good agreement for characteristics such as the plasmon wavelength, simulation and experiment of $Q_{\text {tot }}$ will show more discrepancies because in the simulation: (1) Individual NPs are modeled as cylinders with smooth surfaces, whereas fabricated NPs have some level of surface roughness and are not perfect cylinders. This difference in geometry will affect scattering loss. (2) The dielectric constants used are those measured from Au thin films; ${ }^{30}$ however, Au NPs may have a different imaginary part because the damping of conduction electrons is sensitive to electron scattering, which depends on crystallinity, grain size and the surface area-to-volume ratio of the metal.

Using FDTD calculations, we decomposed $Q_{\text {tot }}$ into $Q_{r}$ (Figure 4C) and $Q_{\mathrm{nr}}$ (Figure 4D) to study how NP height affects the radiative and nonradiative depletion processes. Figure 4C shows that $Q_{r}$ of OLPs is extremely large $(>800)$ at small $k\left(<1.5 \mu \mathrm{m}^{-1}\right)$ for NP arrays with different heights and that $Q_{r}$ decreases with $k$ as the NP height increases. These trends can be explained within the framework of surface Bloch modes. Because the leaky zero-order Bloch harmonic has the same dispersion relation as free space light, the amplitude of $E_{0 \mathrm{z}}$ for the zero-order harmonic can be expressed as $\left|E_{0 z}\right|=\left|E_{0}\right| \cdot|k|$ $(n \cdot \omega / c) \mid$, where $E_{0}$ corresponds to the total electric field for zero-order harmonic. As $k$ approaches 0 , the coupling between the zero-order harmonic and high-order harmonics becomes very weak because $E_{0 z}$ of the zero-order harmonic also approaches 0 . Because the coupling strength of the zeroorder harmonic and higher order harmonics in a grating depends on the amplitude of the periodic modulation, ${ }^{32}$ the plasmon energy stored in the evanescent modes is depleted faster by radiative decay in NPs with larger heights. Figure 4D shows that as the NP height increases, the increase in $Q_{\mathrm{nr}}$ with $k$ becomes less significant. The reason is that NP arrays with larger NP heights support OLPs with stationary character over a broader $k$ range before converging with the $(-1,0)$ RA at high $k$. Therefore, changes in the NP height not only affect $Q_{r}$ by dictating the coupling strength to the leaky zero-order harmonic but also affect $Q_{\mathrm{nr}}$ by modifying the shape of the dispersion relation of OLPs.

In summary, our work has reported a new type of delocalized lattice plasmon in periodic NP arrays. We have clarified the dispersion effects on both far-field and near-field optical properties of delocalized OLPs. We discovered that both radiative and nonradiative processes for energy dissipation are strongly related to the dispersion relation of OLPs, which can be tailored by controlling the NP height. For applications based on delocalized lattice plasmons, therefore, dispersion effects need to be considered to achieve simultaneously narrow resonance linewidths and high local field confinements. We have shown that lattice plasmons combine the advantages of LSPs in isolated NPs (high field confinements) and SPPs on continuous metal surfaces (dispersion tunability); these plasmon excitations offer opportunities from biological and chemical sensing to surface enhanced spectroscopies to photocatalysis.

\section{AUTHOR INFORMATION}

\section{Corresponding Author}

*E-mail: todom@northwestern.edu.

\section{Notes}

The authors declare no competing financial interest.

\section{ACKNOWLEDGMENTS}

This work was supported by the NSF-MRSEC program at the Materials Research Science and Engineering Center at Northwestern University (DMR-1121262) (W.Z., T.W.O.). M.D.H. acknowledges support by the Department of Defense through the National Defense Science and Engineering Graduate Fellowship (NDSEG) Program. W.Z. and M.D.H. gratefully acknowledge support from the Ryan Fellowship and the Northwestern University International Institute for Nanotechnology. This work made use of the NUANCE Center facilities, which are supported by NSF-MRSEC, NSF-NSEC, and the Keck Foundation. Use of the Center for Nanoscale Materials facilities was supported by the U.S. Department of Energy, Office of Science, Office of Basic Energy Sciences, under contract no. DE-AC02-06CH11357.

\section{REFERENCES}

(1) Maier, S. A. Plasmonics: Fundamentals and Applications. Springer: New York, 2007.

(2) Barnes, W. L.; Dereux, A.; Ebbesen, T. W. Surface Plasmon Subwavelength Optics. Nature 2003, 424, 824-830.

(3) Noginov, M. A.; Zhu, G.; Belgrave, A. M.; Bakker, R.; Shalaev, V. M.; Narimanov, E. E.; Stout, S.; Herz, E.; Suteewong, T.; Wiesner, U. 
Demonstration of a Spaser-Based Nanolaser. Nature 2009, 460, 11101168.

(4) Oulton, R. F.; Sorger, V. J.; Zentgraf, T.; Ma, R. M.; Gladden, C.; Dai, L.; Bartal, G.; Zhang, X. Plasmon Lasers at Deep Subwavelength Scale. Nature 2009, 461, 629-632.

(5) Liu, M.; Yin, X. B.; Ulin-Avila, E.; Geng, B. S.; Zentgraf, T.; Ju, L.; Wang, F.; Zhang, X. A Graphene-Based Broadband Optical Modulator. Nature 2011, 474, 64-67.

(6) Nie, S. M.; Emery, S. R. Probing Single Molecules and Single Nanoparticles by Surface-Enhanced Raman Scattering. Science 1997, 275, 1102-1106.

(7) Mühlschlegel, P.; Eisler, H.-J.; Martin, O. J. F.; Hecht, B.; Pohl, D. W. Resonant Optical Antennas. Science 2005, 308, 1607-1609.

(8) Cai, W. S.; Vasudev, A. P.; Brongersma, M. L. Electrically Controlled Nonlinear Generation of Light with Plasmonics. Science 2011, 333, 1720-1723.

(9) Klar, T.; Perner, M.; Grosse, S.; von Plessen, G.; Spirkl, W.; Feldmann, J. Surface-Plasmon Resonances in Single Metallic Nanoparticles. Phys. Rev. Lett. 1998, 80, 4249-4252.

(10) Voisin, C.; Del Fatti, N.; Christofilos, D.; Vallee, F. Ultrafast Electron Dynamics and Optical Nonlinearities in Metal Nanoparticles. J. Phys. Chem. B 2001, 105, 2264-2280.

(11) Wang, F.; Shen, Y. R. General Properties of Local Plasmons in Metal Nanostructures. Phys. Rev. Lett. 2006, 97, 206806.

(12) Christ, A.; Ekinci, Y.; Solak, H. H.; Gippius, N. A.; Tikhodeev, S. G.; Martin, O. J. F. Controlling the Fano Interference in a Plasmonic Lattice. Phys. Rev. B 2007, 76, 201405.

(13) Fedotov, V. A.; Rose, M.; Prosvirnin, S. L.; Papasimakis, N.; Zheludev, N. I. Sharp Trapped-Mode Resonances in Planar Metamaterials with a Broken Structural Symmetry. Phys. Rev. Lett. 2007, 99, 147401.

(14) Hao, F.; Sonnefraud, Y.; Van Dorpe, P.; Maier, S. A.; Halas, N. J.; Nordlander, P. Symmetry Breaking in Plasmonic Nanocavities: Subradiant LSPR Sensing and a Tunable Fano Resonance. Nano Lett. 2008, 8, 3983-3988.

(15) Zou, S. L.; Janel, N.; Schatz, G. C. Silver Nanoparticle Array Structures That Produce Remarkably Narrow Plasmon Lineshapes. J. Chem. Phys. 2004, 120, 10871-10875.

(16) Auguie, B.; Barnes, W. L. Collective Resonances in Gold Nanoparticle Arrays. Phys. Rev. Lett. 2008, 101, 143902.

(17) Chu, Y. Z.; Schonbrun, E.; Yang, T.; Crozier, K. B. Experimental Observation of Narrow Surface Plasmon Resonances in Gold Nanoparticle Arrays. Appl. Phys. Lett. 2008, 93, 181108.

(18) Kravets, V. G.; Schedin, F.; Grigorenko, A. N. Extremely Narrow Plasmon Resonances Based on Diffraction Coupling of Localized Plasmons in Arrays of Metallic Nanoparticles. Phys. Rev. Lett. 2008, 101, 087403.

(19) Vecchi, G.; Giannini, V.; Rivas, J. G. Surface Modes in Plasmonic Crystals Induced by Diffractive Coupling of Nanoantennas. Phys. Rev. B 2009, 80, 201401.

(20) Zhou, W.; Odom, T. W. Tunable Subradiant Lattice Plasmons by out-of-Plane Dipolar Interactions. Nat. Nanotechnol. 2011, 6, 423427.

(21) Kocabas, A.; Senlik, S. S.; Aydinli, A. Slowing Down Surface Plasmons on a Moire Surface. Phys. Rev. Lett. 2009, 102, 063901.

(22) Ochiai, T.; Sakoda, K. Dispersion Relation and Optical Transmittance of a Hexagonal Photonic Crystal Slab. Phys. Rev. B 2001, 63, 125107.

(23) Fan, S. H.; Joannopoulos, J. D. Analysis of Guided Resonances in Photonic Crystal Slabs. Phys. Rev. B 2002, 65, 235112.

(24) Loncar, M.; Nedeljkovic, D.; Pearsall, T. P.; Vuckovic, J.; Scherer, A.; Kuchinsky, S.; Allan, D. C. Experimental and Theoretical Confirmation of Bloch-Mode Light Propagation in Planar Photonic Crystal Waveguides. Appl. Phys. Lett. 2002, 80, 1689-1691.

(25) Ryu, H. Y.; Notomi, M.; Lee, Y. H. Finite-Difference TimeDomain Investigation of Band-Edge Resonant Modes in Finite-Size Two-Dimensional Photonic Crystal Slab. Phys. Rev. B 2003, 68, 045209.
(26) Liu, M. Z.; Lee, T. W.; Gray, S. K.; Guyot-Sionnest, P.; Pelton, M. Excitation of Dark Plasmons in Metal Nanoparticles by a Localized Emitter. Phys. Rev. Lett. 2009, 102, 107401.

(27) Nuzzo, R. G.; Stewart, M. E.; Mack, N. H.; Malyarchuk, V.; Soares, J. A. N. T.; Lee, T. W.; Gray, S. K.; Rogers, J. A. Quantitative Multispectral Biosensing and 1D Imaging Using Quasi-3D Plasmonic Crystals. Proc. Natl. Acad. Sci. U.S.A. 2006, 103, 17143-17148.

(28) Engelen, R. J. P.; Mori, D.; Baba, T.; Kuipers, L. Subwavelength Structure of the Evanescent Field of an Optical Bloch Wave. Phys. Rev. Lett. 2009, 102, 023902.

(29) Zhang, X.; Yang, X. D.; Ishikawa, A.; Yin, X. B. Hybrid Photonic - Plasmonic Crystal Nanocavities. ACS Nano 2011, 5, 2831-2838.

(30) Johnson, P. B.; Christy, R. W. Optical-Constants of NobleMetals. Phys. Rev. B 1972, 6, 4370-4379.

(31) Link, S.; El-Sayed, M. A. Spectral Properties and Relaxation Dynamics of Surface Plasmon Electronic Oscillations in Gold and Silver Nanodots and Nanorods. J. Phys. Chem. B 1999, 103, 84108426.

(32) Zaidi, S. H.; Yousaf, M.; Brueck, S. R. J. Grating Coupling to Surface Plasma-Waves 0.1. 1st-Order Coupling. J. Opt. Soc. Am. B 1991, 8, 770-779. 